\title{
The future of universal access? Merging computing, design and engineering
}

\author{
Simeon Keates, David Bradley, and Andrew Sapeluk \\ School of Engineering, Computing and Applied Mathematics, University of Abertay Dundee, \\ Dundee, Scotland, UK \\ \{s.keates, d.bradley, a.sapeluk\}@abertay.ac.uk
}

\begin{abstract}
Technology is advancing at a fast pace while the shape and nature of computers continues to evolve, with tablets and smartphones illustrating the move away from the traditional notion of a laptop or desktop computer. Similarly, networking and sensing technologies are also developing rapidly and innovatively. All of these technologies have the potential to enfranchise users with severe functional impairments to be better able to control and interact with other people and their surroundings. However, this is only possible if those designing the novel systems based upon these new technologies consider such users' needs explicitly. This paper examines how these technological advances can be employed to support these users in the near future. The paper further discusses issues such as the need for security as systems evolve from control of specific environments to a potential model for interaction in any location.
\end{abstract}

Keywords. Universal access, mechatronics, accessibility, environmental control systems, alternative and augmentative communication (AAC), tablets

\section{Introduction}

Advances in technology have the power to enfranchise users, for example to help them interact with and control their environment. However, technology also has the power to disenfranchise users. If one section of the general population is able to utilize technological developments to perform tasks more efficiently or to access novel services and another section of that same population is not able to do so, then the technology can be considered to have contributed to their individual and collective disability. The power of technology to divide potential users into those who can and those who cannot access a new technology has often been referred to as the "digital divide" [1]. The concept of "universal access" arose from the need to consider the needs of all sections of the population to be able to access new products and services, and thus render the notion of a "divide" as obsolete.

It is worth noting that designers do not begin with the intention of deliberately excluding potential users. Excluded users are excluded customers and, consequently, represent lost potential profit opportunities. Put simply, excluded users will not buy your products and services. So, it is worth exploring the origins of design exclusion.

adfa, p. 1, 2011.

(C) Springer-Verlag Berlin Heidelberg 2011 


\subsection{Universal access and the design of new technologies}

New technologies are often driven by an idea for innovative functionality or features. Once the idea has taken shape, design and development can begin and the race is on to be the first vendor in the marketplace to offer the new product. Being the first to the market is still widely seen as gaining a competitive advantage that can persist for a substantial period of time until other factors, such as lower price or enhanced usability, help other products eat into the market share [2].

In the rush to be first to the market, the overriding imperative is to produce a product that works. It does not necessarily have to work well nor be particularly usable. Consequently, the focus is usually on meeting the engineering challenges first and foremost. However, this is not always the case, for example, the development of the experimental IBM Watson system. Watson was developed to see whether Q\&A technologies had advanced to the stage where such a system could feasibly compete against people in a fairly unrestricted challenge. This culminated in the Jeopardy $!^{\mathrm{TM}}$ challenge [3]. However, while this was a significant engineering achievement in its own right, there was still a strong recognition of the importance of meeting the needs of the end users and this is central to the development of commercial versions of the system [4].

There are other causes of exclusion. For example, sometimes the designers are simply not aware that the products or services that they are developing may be deployed by users who have different functional capabilities. It has been said before that, unless otherwise instructed, designers will often design for themselves [5]. In other words, they will typically envisage the user as possessing the same capabilities, knowledge and experience as themselves. This is only human nature - we know ourselves better than anyone else. The full range of reasons for design exclusion and methods for countering that exclusion are considered elsewhere [6]. However, they can be summarized as recognizing the need to explicitly consider the needs of all users through the whole product lifecycle - from initial design to final decommissioning [2] - and how advances in technology offer both potential opportunities for, and challenges to, access to new products and services.

With this perspective firmly in mind it is timely to consider how recent advances in mobile, sensor and communications technologies can offer increased opportunities for universal access. One area where these technologies can combine to offer new opportunities is that of environmental control systems.

\section{Environmental control / home automation systems}

Environmental control systems (ECS) and home automation systems (HAS) allow users with severe functional impairments to carry out tasks such as switching on lights or changing the temperature in their house [7]. For the purposes of this paper, we will use ECS to describe both types of systems.

Users of such systems might typically be wheelchair users and experience limitations such as restricted range of motion, spasms, tremor and reduced strength. They may have conditions such as cerebral palsy or muscular dystrophy. As such, they typically find 
using many domestic devices, such as light switches and heating controls, almost impossible to use unless they have been especially adapted to their personal needs. Environmental control systems remove the need for the users to reach light switches on a wall, by allowing remote control of these devices from their wheelchair.

Early systems typically used infrared technology - such as found in the typical television remote control - to control transducers embedded in each device intended to be controlled and had a very simple architecture [8]:

1. The central processing unit (CPU) - the core of the system that processes the user commands and transmits the control signals, typically from switches, to the corresponding peripheral devices

2. The visual display - to show the user the environmental functions and peripheral devices that are available

3. The transducer - the control switch used to register the user's input action and convert it to a control signal that is sent to the CPU

4. The peripherals - the devices embedded in the environment that are to be controlled through the environmental control system via automated actuators

The basic underlying architecture of environmental control systems has not changed substantially since the mid 1980s when these systems began to appear commercially. However, the enabling technologies available have changed substantially. Rather than viewing components such as the CPU, visual display and transducers as separate entities, the advent of more powerful environmental control systems in recent years has allowed these features to be co-located in a single unit. Similarly, trends are moving towards viewing the context of use as not simply a house with a few automated devices, but, increasingly, a smart environment offering ambient intelligence [9].

Furthermore, developments in mainstream computing now raises the interesting prospect of such systems being controlled by a simple app on an off-the-shelf device such as an Appe iPad or Samsung Galaxy. Similarly, other communication technologies than simple infrared beams mean that it is possible to control devices in the home from ever increasing distances. The remainder of this paper will look at those technologies and the implications for the design of the environmental control systems of the near future.

\section{Communication, location and control technologies}

There are several communications and location technologies available that can be potentially used for controlling an ECS.

\subsection{Communication technologies}

The following is an indicative list of the range of communication technologies that can be used: 
1. Infrared (IR) beams. These are the traditional communication technology of choice for controlling devices in the home. They have low power consumption, meaning excellent battery life. However, they only offer strictly "line-of-sight" control and have limited range $(<10 \mathrm{~m})$, meaning that their range is limited to devices within the user's immediate proximity. They also offer very little security as there is no command authentication required, i.e. there is no requirement for a password nor for the device sending the command codes to be recognized or identified. As long as the correct command codes are received, then the environmental control system will respond to the command, regardless of the source of origin of the command.

2. Radio Frequency (RF) controllers. These work on the same principles as IR ones, but with the difference that radio waves are capable of penetrating through some solid matter. The greater the power used, the greater the penetration. Consequently, RF controllers offer more flexibility in use, through a longer range and relaxing the line-of-sight requirement. However, security is still comparatively weak.

3. Bluetooth. Extending the control range a little further, Bluetooth can be used to transmit control commands up to 100 metres. Bluetooth has a major advantage in being widely available through many devices, especially mobile and smartphones. It also has good security potential if set up correctly. However, if left unprotected, the ubiquity of Bluetooth controllers is a potential disadvantage, as it would become quite straightforward to penetrate an open network. Bluetooth also suffers from more substantially power requirements, and thus decreased battery life, than many of the alternative technologies.

4. Low-rate wireless personal area networks (LR-WPANs). The IEEE 802.15.4 standard [10] offers digital radio control of devices up to 1 mile apart, which is a significant step in increasing the potential control range of an ECS. LR-WPANs also offer a substantial increase in security, supporting the use of cryptographic methods to ensure point-to-point communications. However, such networks are designed to support only small, infrequent packets of data rather than more substantial volumes. Consequently, while they could be used to switch lights on, for example, they would most likely struggle to support a live video feed.

5. Global System for Mobile Communications (GSM). The next increase in the distance over which an ECS can be controlled is to move from point-to-point personal network connection to using established networks and submitting the control commands securely via those. One example of this is sending SMS control messages via the GSM mobile phone networks. Use of the GSM networks allows an ECS to be controlled from anywhere in the world where there is a usable phone signal. However, such messages can suffer from significant delays in their delivery and sometimes even non-delivery. This question mark over the reliability of delivery of the commands is a significant problem with GSM-based control. Security is typically achieved by requiring authentication codes in the SMS messages sent. These codes, or keys, can be of any length and so offer a reasonable amount of security.

6. Internet. An alternative solution that also offers global reach is to control the ECS via Internet technologies. Wi-Fi connections are available in many buildings and mobile phone networks typically support 3G or 4G Internet connectivity in many metropolitan areas. While such connectivity is not as geographically widespread as, 
say, GSM coverage, Internet connections offer a far greater bandwidth for communication, potentially supporting live video feeds with comparative ease and also offering access to typical Internet security protocols. Arguably the biggest disadvantages are the power requirements to maintain such a connection leading to short battery life compared with, for example, an IR remote control and also that network coverage, while growing continually, is still far from universal.

With the potential increase in range of the new communication technologies from line-of-sight, where the user would have to be within the house, to potentially global, it is helpful to be able to identify where the user is. This knowledge can be used to tailor the functions available to the user - effectively there can be a set of "remote" functions and a set of "at home" functions. There are different technologies that can be used to establish the location of the user.

\subsection{Location technologies}

There are two principal location technologies of interest here:

1. Radio Frequency Identification (RFID) tags - RFID tags act more as a proximity detection system rather than a location detection one. Effectively, they can be used to establish that a tagged object is within a particular distance of the sensor or reader. That distance can be up to 200 metres, depending on the output power of the tags. The advantage of RFID tags is that an independent local proximity detection network can be established.

2. Global Positioning System (GPS) - GPS is the system used in many navigation aids. It uses the triangulation of signals from a network of satellites to establish in objects location and even its velocity. As its name suggests, a GPS-enabled device is capable of establishing its location anywhere in the world. However, it does have substantial power demands and can deplete batteries in a matter of hours. Essentially an outdoor technology requiring a line-of-sight to satellites, it can be confused by geographical features, such as tall buildings and mountains, thus limiting the data resolution that can be obtained.

There is in addition a third possible approach:

3. Local sensor network - Significant research has been performed towards developing ambient intelligent environments [9]. This research facilitates the development of more ad hoc networks of sensors that can also locate the position of a user within a space. These sensors could range from simple passive infrared (PIR) sensors to sophisticated computer vision systems, where a camera is used to find the user within the living space. Such sensor networks, especially those based on vision techniques, can be very powerful offering the ability to detect whether a user has fallen down, has been inactive for an extended period of time or is showing signs of emotional distress. However, they are still somewhat experimental, expensive to install and only work within the limited distance range of the sensors. 
With an understanding of these technologies, it is possible to begin to see how new environmental control systems could be designed and how their functionality can be extended from simply switching lights and domestic appliances on and off to offering a more comprehensive ability for a user to control his or her environment.

\subsection{Control technologies}

Typically, environmental control systems are operated via a special purpose control unit designed specifically to interact with control actuators on each of the domestic appliances that are to be controlled. The advantage of this approach is that the risk of incompatibilities between all of the components is virtually eliminated. It also facilitates the development of custom interfaces to meet the specific needs of an individual user as the transducers can be selected to meet the movements that are easiest and most reliable for that user. For example, these could include binary switches, electromyogram (EMG) sensors, etc.

However, such customized controllers are often expensive and do not always support any functionality beyond control of the identified peripherals. So, they do what they are designed to do and do it well, but if the user wants to add new functionality or to interact with another environment, then those controllers are most likely not going to be suitable for that.

This is where the rise of the new generation of ultra-mobile computing technology raises intriguing possibilities. Tablet computers, such as the Apple iPad and the Samsung Galaxy, are increasing in raw computational power and capability. They are small and lightweight and could fit easily onto a wheelchair in the same way that many special purpose alternative and augmentative communication (AAC) devices have done for a number of years.

These new tablet computers are already designed to interact with domestic appliances, such as televisions, and can even replace devices such as the telephone by using Voice over Internet Protocol (VoIP) calling services. They are straightforward to connect to the Internet and many also offer access to cellular data networks.

The new tablets offer a new model for software development and deployment. The ability to download and install small, agile applications (apps) quickly and easily allows the tablets to deliver highly customizable interaction opportunities to the users. A number of commercial home automation apps are already available for tablets, typically based on the Universal Powerline Bus (UPB) standard, where control signals are sent over a building's existing electricity cables [11] or where the intended peripheral is independently TCP/IP enabled, i.e. is capable of being connected to the Internet.

It is not difficult to envisage how using such a device to control an ECS could lead to interesting possibilities for improving a user's ability to not only interact with and control their local domestic environment, but also provide a more generalized and universal ability to interact with a wider range of environments and systems from an increased spread of geographical locations. Such systems go beyond the concept of traditional environmental control systems. For our purposes here, we will refer to these new, more powerful systems as unified control systems (UCS). 


\section{A new model of unified control systems}

As discussed in the preceding section, apps already exist for using a tablet to control home automation / environmental control systems. Similarly, AAC apps have been developed to allow the use of tablets to be used for interpersonal communication for users with difficult speaking ${ }^{1}$. Many tablets are already GPS-enabled, providing location detection and they all provide access to the Internet, either through Wi-Fi connectivity or mobile phone data networks.

Thus it can be seen that the elements for a single, combined "unified control system" are largely in place. It could be argued that what is needed now is a framework for bringing this disparate functionality into a cohesive structure. Rather than simply focusing on getting the existing apps to "talk to each other," it is worth examining a variety of possible use-case scenarios to see how a unified control system might be structured.

\subsection{Example use-cases}

The following are a few example use cases for unified control systems, These illustrate how an appropriately designed control system, based on general standards, can significantly improve the independence of a user with a severe motor impairment:

1. Remote control of the house. This is perhaps the most obvious use case. Most environmental control systems are designed to support control of the domestic appliances within a home when the user is physically located there. Home automation systems are intended to support remote control of those systems. The unified control system should support both of these options and offer the ability to autonomously control the functions of the house (such as turning on the heating if the temperature falls too low) and to allow the user to customize all of the automated features of the house at will.

2. House access control. A fully automated house should include a capability to "centrally lock" the house, not unlike how remote central locking is available on most cars. A simple extension of this model, though, is to offer central or selective unlocking. A suitable enabled unified control system should be able to identify when the user is approaching the house and automatically unlock and/or open the front door to allow the person to enter the house without having to issue an explicit command. One possible method for achieving this would be to use GPS location to establish when the user is within the grounds of the house. Additionally, the users should be able to use the cameras within the house to securely grant access to repair personnel and the like. Such permission may require explicit confirmation commands by the user - i.e. "Should this person be granted access to the house?"

3. Access to other smart environments. If the unified control system uses widely accepted standards, then it should be possible to allow the user to interact with and

\footnotetext{
${ }^{1}$ Examples of some of the AAC products available can be found at: http://www.friendshipcircle.org/blog/2011/02/07/7-assistive-communication-apps-in-the-ipad-app-store/
} 
control any other smart environment that they enter. Such functionality would offer a significant increase in their level of independence as they would be offered the same level of personalized control of their general environment as they would have in their own home.

4. Inter-personal communication. If a tablet computer is used as the controller, it would be straightforward to add AAC functionality to allow it to operate as a text-to-speech device. It is also possible to couple that to VoIP services, say, to provide a fully accessible telephone capability within the one device.

5. Areas of life endeavour. Keates et al. [12] proposed 5 areas of life endeavour for users with severe functional impairments that computer systems should focus on supporting. These included: lifelong learning and education; workplace; real world (extended activities of daily living); entertainment; and, socializing. Looking at the first of these, it is straightforward to envisage how a unified control system should be able to control the user's workplace as well as their domestic environment.

6. General computer use. A tablet computer is capable of supporting Internet access, personal entertainment and many other functions. These could be embedded in and supported by a general user interface (UI) structured to meet the functional capabilities of the user.

\subsection{Examples of the technology in practice}

The School of Engineering, Computing and Applied Mathematics (SECAM) at the University of Abertay has been researching methods of developing smarter environments and enabling users to interact with and control those environments. There are 2 example systems that are of particular interest here. Both systems were exhibits in the "Robotics" exhibition hosted by "Sensations", the Dundee Science Centre, in the summer of 2012:

1. The GSM-enabled doll's house. A custom-built doll's house has been installed with a GMS receiver and actuators on the doors, lights and windows (see Figure 1). Visitors to the "Robotics" exhibition were invited to send SMS control messages, consisting of 10 different hexadecimal commands codes, to the house to see the effects of the actions. The house has since been enhanced to include Wi-Fi and support commands from a graphical user interface. The next stage of development is to add additional features to the house, including embedded sensors and webcams to support more typical domestic operations. A smart meter will be added, so users can monitor the power consumption of the house remotely, including the effects of running different domestic appliances on the house's overall energy consumption. 


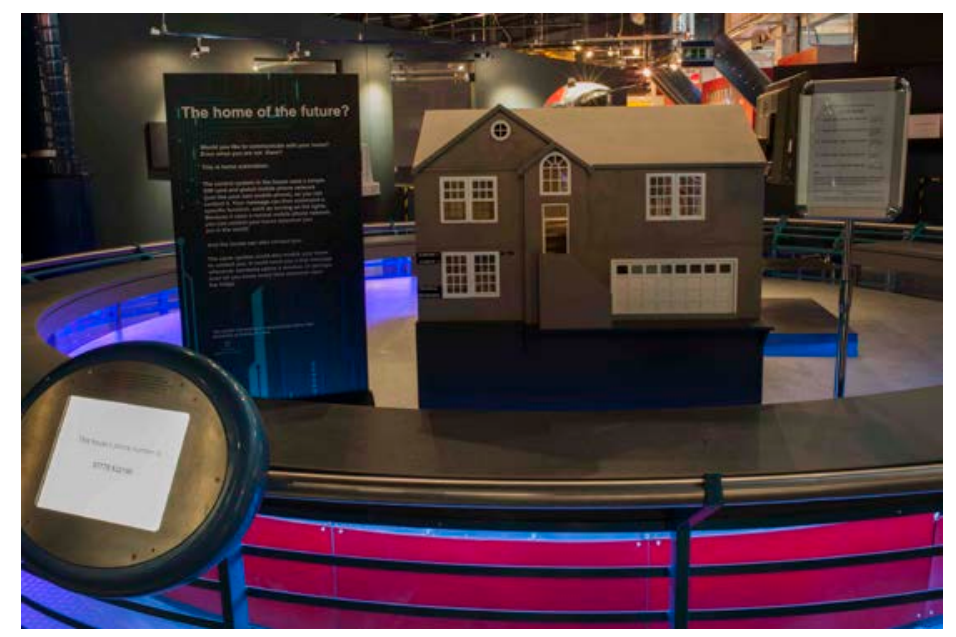

Figure 1. The GSM controlled doll's house. This photograph was taken at the Robotics exhibition hosted by the Dundee Science Centre in the summer of 2012.

2. A mobile personal communication device. A remote chat-bot was installed in a model robot dog (see Figure 2). The chat-bot was controlled via a regular touchscreen, which would be straightforward to substitute with a tablet. The system demonstrated how easy it is to operate a complex chat-bot on a device with limited local capability, but with an Internet connection. The chat-bot processing was performed on remote servers, minimizing the need for local processing power. The next stage of development is to make the dog autonomously mobile, allowing it to follow the user to provide more mobile communication functionality.

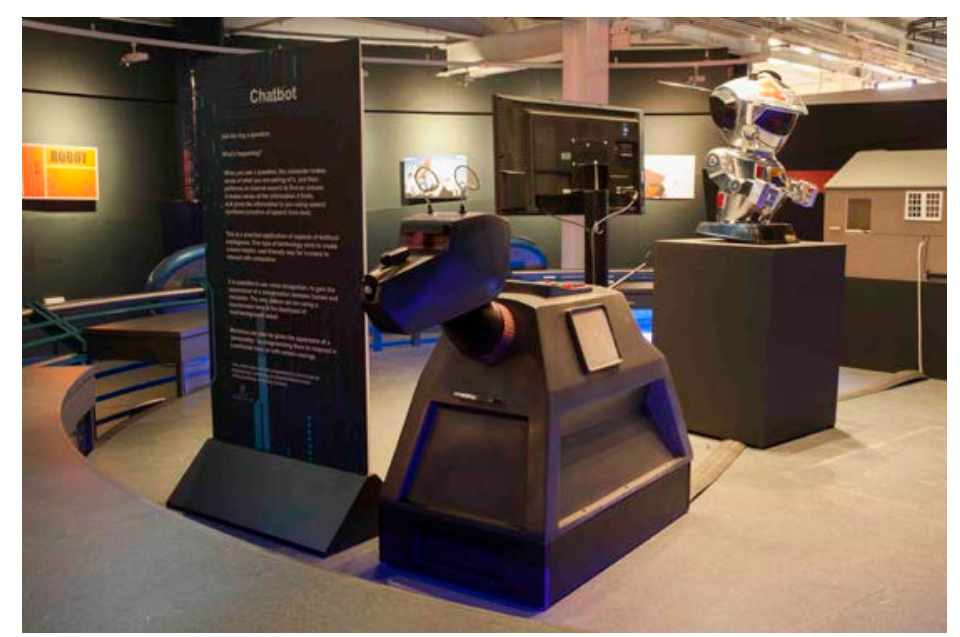

Figure 2. A chat-bot in a robotic dog. The next stage of development is to add more functionality to make an autonomous mobile communication device. 


\section{Conclusions}

This paper has examined possible developments for supporting users with severe motor function impairments by examining how existing technologies can be brought together in innovative ways to support the interaction with and control of a wider set of environments and contexts of use. The technologies required to facilitate the development of such a system largely already exist. What is required is a suitable framework for bringing these capabilities together into a cohesive user experience, ideally through a single user interface. The next steps for this research team will be to develop the existing prototypes further to examine how such a framework could be developed.

\section{References}

1. Pieper, M.: Digital divide and learning disabilities: counteracting educational exclusion in information society. ACM SIGACCESS Accessible Computing. 83, 37-41 (2005)

2. Keates, S.: Designing for accessibility - a business guide to countering design exclusion. CRC Press (2006)

3. Keates, S., Varker, P., Spowart, F.: Human-machine design considerations in advanced machine-learning systems. IEEE/IBM Journal of Research and Development. 55(5), 4:1-4:10 (2011)

4. Keates, S., Varker, P.: The game is afoot, Watson: DeepQA systems and the future of HCI. In: Rebelo, F., Soares, M.M. (eds.) Advances in Usability Evaluation Part II, pp. 341-348. CRC Press, Boca Raton, FL (2012)

5. Cooper, A.: The inmates are running the asylum: why high tech products drive us crazy and how to restore the sanity. SAMS Publishing, Indianapolis, USA (1999)

6. Keates, S., Clarkson, P.J.: Countering design exclusion - an introduction to inclusive design. Springer-Verlag: Heidelberg (2003)

7. Dickey, R.: Electronic technical aids for persons with high level spinal cord injury. Central Nervous Systems Trauma. 3(1), 93-110 (1986)

8. Dickey, R., Shealey, S.H.: Using technology to control the environment. American J of Occupational Therapy. 41(11), 717-721 (1987)

9. Emiliani, P.L., Stephanidis, C.: Universal access to ambient intelligence environments: Opportunities and challenges for people with disabilities. IBM Systems Journal. 44(3), 605619 (2005)

10. IEEE: IEEE Standard P802.15.4/D18, Low Rate Wireless Personal Area Networks (2003)

11. Powerline Control Systems: Universal Powerline Bus - The UPB System Description. (2003) Available at: http://www.smarthomeusa.com/Common/UPB/UPBdescription.pdf

12. Keates, S., Kozloski, J., Varker, P.: Cognitive impairments, HCI and daily living. LNCS, Vol. 5614, Universal Access in Human-Computer Interaction. Addressing Diversity. Pp. 366-374. Springer-Verlag: Heidelberg (2009) 